\title{
Study of the degree of disability in children suffering from Hemophilia- A using PedHAL score in south Gujarat area
}

\author{
Vijay B Shah ${ }^{1}$, Kirti Mehta $^{2 *}$, Silky Agrawal ${ }^{3}$ \\ ${ }^{1}$ Professor and Head, ${ }^{2}$ Associater Professor, ${ }^{3}$ Ex-resident, Dept. of Pediatrics, Government Medical College, Surat, Gujarat, India
}

*Corresponding Author: Kirti Mehta

Email: drkirtimehtagamit@gmail.com

\begin{abstract}
Introduction: The severe forms of hemophilia are characterized mainly by frequent haemarthroses leading to chronic crippling haemarthropathy when not treated very early or prophylactically. The occurrence of disability in developing and resource limited country like India is more common and severe as compared to those countries where prophylactic treatment home based factor therapy is available. PedHAL Score helps in assessment of disability in paediatric population based on activities of daily living.

Objectives: the objective of the study was to find out the degree of disability in children (4-14 years) suffering from hemophilia in South Gujarat region using PedHAL score. The other objectives were to assess the effect of severity of disease on degree of disability in children suffering from hemophilia and to assess the effect of different factors (therapeutic and demographic) on degree of disability.

Materials and Methods: The study was conducted among the pediatric hemophilia patients between 4 to 14 years of age group. A predesigned questionnaire Pediatric hemophilia activity list (PedHAL) parent version 0.11 was selected to measure the impact of hemophilia on daily life activities as perceived by child's parent. The PedHAL is pediatric version, to assess functional abilities in children, to measure the impact of hemophilia on self-perceived functional abilities in children. The median PedHAL total score was 100 with Inter Quartile Range of 85 to 100 .

Results: Based on the median value the worst affected function are sitting, kneeling and standing (median 94) followed by other functioning of leg. (median 94.55). Mean PedHAL Score was slightly lower in illiterate cases (83.1) compare to cases educated up to primary (86.55). Mean PedHAL Score was 86.44 in severe cases and it was 74.17 in moderate cases. Mean PedHAL Score was slightly higher in cases having more than 20 bleed till date (88.87) compare to those having less bleeds (87.876). (p<0.05) Mean PedHAL Score was higher in cases receiving only factor 8 (96.95) compare to cases receiving factor 8 with other drugs (81.45).

Conclusion: The mean PedHAL score is not associated with age of the patient; nutritional status of patient; education of patient; severity of disease; number of bleeds till date; distance from home to hospital; habit of exercise; regularity of exercise; type of family of patient; family history of hemophilia; education of patients' mother; and family income of the patient. Early onset of symptoms is associated with lower PedHAL score and thus higher disability. Cases having 6 or more bleed per year had lower PedHAL score and thus higher disability. Higher PedHAL score and thus lower disability is associated with cases taking only factor 8 compared to cases taking other medication along with factor VIII.
\end{abstract}

Keywords: Hemophilia A, Degree of disability, Pediatric hemophilia activity list (PedHAL).

\section{Introduction}

Hemophilia is a group of $\mathrm{X}$-linked bleeding disorders due to deficiency of clotting factors VIII (Hemophilia A) and IX (Hemophilia B). The clinical manifestations of hemophilia A and hemophilia B are indistinguishable, and occur in mild, moderate, and severe forms. They are the only blood clotting disorders inherited in a sex-linked pattern. The severe forms of hemophilia are characterized mainly by frequent haemarthroses leading to chronic crippling haemarthropathy when not treated very early or prophylactically. The occurrence of disability in developing and resource limited country like India is more common and severe as compared to those countries where prophylactic treatment home based factor therapy is available. Highly purified concentrates, prepared from human plasma and manufactured by recombinant technology, are available for treatment and are considered to be safe and effective. The main complication of treatment is the development of antibody inhibitors against either factor VIII or factor IX, which are more common in patients with hemophilia A than in patients with hemophilia B. ${ }^{1,2}$

In 2009, the World Federation of Hemophilia (WFH) has identified 1,53,251 people with hemophilia throughout the world. ${ }^{3}$ Seventy-five per cent of the global hemophiliacs live in developing countries, where probably only one in five cases is diagnosed, and there is little or no care available. ${ }^{4}$ Hemophilia as a disease and its management has a large impact on the community, including social integration and economics. Inability to be an active part of society and high cost of the medical care make this disease an important problem for all haemophilic patients.

In developed countries, early treatment of bleeding episodes and home therapy quickly evolved as the primary management option. Presence of specialized interdisciplinary centres, training and education of patients are the main advantages of the comprehensive treatment concept in developed countries.

This is not the case in most developing countries like India where the government does not have the resources to buy the necessary quantities of coagulation factors in the face of more urgent health priorities and hardly any patients can afford to pay for their own treatment even for ondemand home therapy. Other problems are insufficient supply with clotting factor concentrates, lack of access to these concentrates and absence of specialized care centres. ${ }^{5}$ Hemophilia being a life long disabling disease without prophylactic treatment, as in developing country like India, 
many children with severe hemophilia suffers from recurrent bleeding episodes of major joints. Eventually these bleeds will result in irreversible joint damage and limitation in activities of daily living. In the WHO's International classification of Functioning, Disability and health (ICF) ${ }^{4}$ activities in daily life are recognised as an important link between structural problems, participation and quality of life. PedHAL Score helps in assessment of disability in paediatric population based on activities of daily living. ${ }^{6}$

There has been no previous study on the disability and its comparison with other relevant demographic factors in pediatric hemophilia patients in the South Gujarat region. This study was designed to determine the existing pattern of disability and association with various demographic factors in the region. The study also designed to understand principle domains of activity limitation among the hemophilia patient on replacement therapy. Along with this the idea was to provide insight into the need of prophylactic treatment for prevention of disability among hemophilia patients to help stakeholders involved in hemophilia care, such as providers, decision- and policy-makers identify and address the needs of people with hemophilia. At the same time, the methods employed may have broader applications in research for other chronic, rare diseases.

So, the objective of the study was to find out the degree of disability in children (4-14 years) suffering from Hemophilia in South Gujarat region using PedHAL score. The other objectives were to assess the effect of severity of disease on degree of disability in children suffering from hemophilia and to assess the effect of different factors (therapeutic and demographic) on degree of disability.

\section{Materials ad Methods}

This was a cross-sectional study conducted at the tertiary care teaching hospital that caters mainly urban, peri-urban and rural population of South Gujarat. The total duration of study was approximately one and half year.

The study was conducted among the pediatric hemophilia patients attending the hemophilia day care centre. As this study involve pediatric age group and special vulnerable group all care was taken to protect ethical rights of the patients. An approval was sought from the institutional ethical committee before the start of the study. For the eligible children parents/guardian were informed in detail about the study and its process. They were also explained about probable benefits and potential risk associated with the study in vernacular language. Then after, a written consent was taken in vernacular language. In case the parent or guardian was illiterate the consent form was filled up in present of literate witness. A high degree of confidentiality was maintained at all level from recruitment to data collection to analysis.

Inclusion criteria for the enrolment of subject were

1. Already diagnosed cases of hemophilia A

2. Aged- 4 completed years upto 14 completed years

3. Gave the voluntary informed written consent (from parents or guardian).
Subjects not fulfilling the inclusion criteria were excluded from the study.

Considering the prevalence of hemophilia ( 1 case per 10,000 Population), there are 84 cases enrolled of age group 4-14 years in the South Gujarat area as per data in hemophilia day care centre. However, only 51 patients were able to be included for statistical analysis.

For all eligible case a predesigned questionnaire Pediatric hemophilia activity list (PedHAL) parent version (0.11) was selected to measure the impact of hemophilia on daily life activities as perceived by child's parent. The questionnaire was designed by Groen W \& Van der Net J et al. and posted in world federation of hemophilia official website. [7] One of the Parents of children aged 4-14 years known case of hemophilia A were given the parent version of Ped HAL score 0.11 were explained each questionnaire in their local language and response was recorded for each activity as reported by parent. It took around ten minute for parent to complete the questionnaire. Parents were interrogated in their regional language the questionnaire, also for comparison with relevant demographic factors a separate questionnaire was prepared.

The Hemophilia Activities List score its pediatric version is used to measure the impact of hemophilia on selfperceived functional abilities in children. The current version assesses the same seven domains as the HAL.

The PedHAL should be selected to measure the impact of hemophilia on self-perceived functional abilities in children.

The current PedHAL version (0.11) consists of 53 items in seven domains:

1. Sitting/kneeling/standing (10 items)

2. Functions of the legs (11 items)

3. Functions of the arms (6 items)

4. Use of transportation (3 items)

5. Self care ( 9 items)

6. Household tasks (3 items)

7. Leisure activities and sports (11 items)

A parent/proxy (age 4-8) and child version (8-18) was constructed with some minor linguistic differences.

Psychometric properties of the score:

1. Construct validity (preliminary data of pilot test)

Convergent validity: Most subscales showed moderate associations with joint examination $(\mathrm{r}=0.42-0.63)$ and moderate to good associations with the physical function subscale of the Child Health Questionnaire (CHQ-50) $(\mathrm{r}=0.48-0.78)$.

Divergent/discriminant validity: Preliminary analysis shows the pedHAL does measure functional abilities. No significant associations were found between the pedHAL and the subscales mental health and behaviour, except for the subscales leisure and sport and mental health $(\mathrm{r}=0.47)$.

2. Criterion validity has not been assessed. There is no gold standard to compare to the PedHAL.

3. Reliability (preliminary data of pilot test): Reproducibility, as assessed by a Wilcoxon signed rank order test, showed no differences on the level of subscales 
and sum score of the PedHAL on test and retest for both parent and child forms.

The limits of agreement (LOA) were low on most subscales for the parent (LOA $0.2 \pm 0.9$ ) and child (LOA 0.2 \pm 0.9 ) forms.

\section{ADMINISTRATION of score:}

1. Time to complete: 10-20 minutes for both the child and parent versions. This study makes use of parent version of score only to assess disability.

2. Equipment/space required: None. Only the questionnaire and are pen are needed.

3. Training required: None. The instructions provided are self-explanatory. Explained in

4. Cost: None

5. Scoring/scaling/interpretation of results: Normalized scoring per domain and sum score, analogous to that of the Hemophilia Activities List (HAL). A detailed scoring system is available in the pedHAL version 1.0.

After getting the score, it was correlated with various demographic and medical parameters of the participants.

\section{Results}

Current study was done on hemophilia A patients of age group 4-14 years that visited hemophilia day care centre of surat city for treatment. Study included 51 hemophilia A patients.

PedHAL score parent version 0.11 developed by Groen W, Van der Net J, Helders P, Fischer K. and published in world federation of hemophilia guideline for assessment of activity limitation in hemophilia children was utilized to find the disability score among the participants. ${ }^{7}$ The score consist of seven different activity domains with total of 53 items. A raw score is converted into a normalized score that ranges from 0 (worst functional status) to 100 (best possible functional status). For the current study population as shown in table 1, the median total score was 100 with Inter Quartile Range of 85 to 100 .

Table 1 shows domain wise disability in hemophilia patients. Based on the median value the worst affected function are sitting, kneeling and standing (median 94) followed by other functioning of leg. (median 94.55). This suggest that problems are more in functions involving lower limb joints. The third affected domain was Leisure activities and sports activities. The remaining domains were also affected but their lower IQR is above 90. According to current study $74.5 \%$ of study population was disabled (PedHAL score <100), while only remaining $25.5 \%$ was disability free (PedHAL score 100) as shown in table 2. The table shows that out of total 51 cases, 38 cases had disability in one or more domain. Highest number of disable was found in functioning of Leg (domain 2) where $64.7 \%$ cases had varied level of disability. Domain 1 (Sitting/kneeling/standing function) was the second most affected domain where $60.8 \%$ cases had disability. Least disability was found in household task.

The response of parent was considered not applicable if the child had not been allowed or had ever performed the activity, such items were considered invalid. In current study it is also shown in table 2. Highest invalids were found in Leisure activities and sports (domain 7) where $70.1 \%$ items were not able to score. This is followed by use of transportation (domain 4) and household task (domain 6). Least invalids were in domain 1 . Table 3 shows the distribution of all participants according to their demographic data. The table also shows the correlation of various demographic parameters with the total PedHAL score. Age wise distribution of the cases indicates that 24 $(47.1 \%)$ patients were in age group of 10-14 years, 21 $(41.2 \%)$ patients were in age group of 6-9 years and 6 (1.8\%) patients were in 4-5 years of age. Mean PedHAL Score was highest (93.02) in 4-5 year of age group while it was lowest (84.01) in 10-14 year of age.

Nutritional status according to BMI indicates that 13 (25.4\%) cases were underweight, 25 (49\%) cases were normal weight, $5(9.8 \%)$ cases were overweight, and 8 $(15.7 \%)$ were obese. As shown in the table mean PedHAL Score was lower in underweight cases (81.18) compare to normal weight cases (89.96). Application of statistical test indicates that the difference was statistically non significant. ( $>0.05)$ Similarly the correlation of normal weight with the overweight + obese participants was non-significant. $(\mathrm{p}>0.05)$

As shown in above table out of 51 cases of hemiphilia 8 (15.7\%) were illiterate while remaining 43 (84.3\%) were studied up to primary school. Correlation with the Ped HAL score of different group according to literacy difference was statistically not significant $(\mathrm{p}>0.05)$. Education of parents shows that $11(21.6 \%)$ fathers were illiterate or studied up to primary or below. And father of $40(78.4 \%)$ cases were studied up to secondary school or above. While mother of $27(52.9 \%)$ cases were illiterate or studied up to primary or below. And mother of $24(47.1 \%)$ cases were studied up to secondary school or above that. PedHAL Score was higher in cases whose mother were educated up to primary or below (88.65) compare those who educated up to secondary school or higher (87.01). PedHAL Score was higher in cases whose mother were educated up to primary or below (88.65) compare those who educated up to secondary school or higher (87.01). However, Application of statistical test indicates that this difference was statistically not significant $(p>0.05)$. While the difference in PedHAL score of father's education is statistically significant $(p<0.05)$

As seen in the table, out of 51 cases of hemophilia 41 $(82.4 \%)$ were residing in nuclear family while remaining 9 $(17.6 \%)$ were staying in joint family. PedHAL score was not correlated with the type of family patient. $(\mathrm{p}<0.05)$. Total $38(74.5 \%)$ cases were residing $15 \mathrm{~km}$ or less from the hospital while remaining $13(25.5 \%)$ cases were residing more than $15 \mathrm{~km}$ away from the hospital. Table shows that mean PedHAL Score was higher in cases residing more than $15 \mathrm{~km}$ away from the hospital (89.08) compare to cases residing near to that. However, application of statistical test indicate that this difference was statistically not significant $(\mathrm{p}>0.05)$, so PedHAL score was not correlated with the distance from home to hospital. 
As shown in the table out of 51 cases of hemophilia 27 $(52.9 \%)$ were having habit of exercise while remaining 24 $(47.1 \%)$ were never do exercise. Among those who were having habit of exercise, $17(63 \%)$ patients do exercise regularly and remaining 10 (37\%) were not doing exercise regularly. The table shows income distribution of cases according to their family income. Based on the modified Prasad's classification five income category were made. Highest cases were from middle income and upper middle income group. As shown in the above table mean PedHAL Score was increasing with decrease in income. Application of statistical test also indicate that this difference was statistically significant $(\mathrm{p}<0.05)$.

As seen in the table 4, $23(45.1 \%)$ cases had age of onset was below 12 months while in remaining 28 (54.9\%) cases the disease was started at age of 12 month or later. PedHAL Score was lower in cases developed symptoms before the age of 12 months (78.75) compares to cases that developed it later (95.37). And the difference was statistically significant. $(p<0.001)$ The history of bleeding shows $46(84.3 \%)$ were having less than 6 bleed in last one year while $8(15.7 \%)$ were having 6 or more bleed in last one year. While total bleeding episode till date shows 31 $(60.8 \%)$ were having 20 or less bleed till date while 20 $(39.2 \%)$ were having more than 20 bleed till date. PedHAL Score was slightly higher in cases having more than 20 bleed till date (88.87) compare to those having less bleeds (87.876) but it was not significant. ( $>>0.05)$ However, PedHAL Score was higher in cases having less than 6 bleed per year (91.02) compare to those having 6 or more bleeds (91.02). ( $\mathrm{p}<0.05)$.

Family history was present in $24(47.1 \%)$ cases while in remaining $27(52.9 \%)$ cases there was no history of hemophilia. PedHAL Score was only slightly higher in cases with family history of hemophilia (88.25) compare to cases without having family history of hemophilia (84.02) which was statistically not significant. $(p>0.05)$ The table number 4 shows that 51 cases of hemophilia A 2(3.9\%) were having mild disease, $4(7.8 \%)$ were having moderate disease while remaining $45(88.2 \%)$ were suffering from severe form of the disease. PedHAL Score was marginally higher in severe cases (86.44) compare to moderate cases (74.17). However, difference was statistically not significant ( $p>0.05$ ) Out of 51 cases of hemophilia, 15 (29.4\%) were receiving only factor 8 as treatment while remaining 36 (70.6\%) were receiving CRYO and FFP along with factor 8 . PedHAL Score was higher in cases receiving only factor 8 (96.95) compare to cases receiving factor 8 with other drugs (81.45). Application of statistical test indicate that this difference was statistically significant. $(\mathrm{p}<0.01)$

Table 1: Domain wise score and affected individual

\begin{tabular}{|l|c|c|c|c|}
\hline Domain & Median* & IQR\# & Minimum & Maximum \\
\hline Sitting/kneeling/standing & 94 & $84-100$ & 4 & 100 \\
\hline Functions of the legs & 94.55 & $74.29-100$ & 15 & 100 \\
\hline Functions of the arms & 100 & $96.67-100$ & 50 & 100 \\
\hline Use of transportation & 100 & $100-100$ & 6.7 & 100 \\
\hline Self-care & 100 & $92.50-100$ & 33.3 & 100 \\
\hline Household tasks & 100 & $100-100$ & 40 & 100 \\
\hline Leisure activities and sports & 100 & $88.75-100$ & 20 & 100 \\
\hline Total Score & 100 & $85-100$ & 0 & 100 \\
\hline
\end{tabular}

$* 50^{\text {th }}$ Observation \#Inter Quartile Range $\left(25^{\text {th }}\right.$ to $\left.75^{\text {th }}\right)$

Table 2: Domain wise number of disable and their percentage

\begin{tabular}{|c|c|c|c|c|c|c|c|}
\hline \multicolumn{2}{|r|}{ Domain } & $\begin{array}{l}\text { Total } \\
\text { Items }\end{array}$ & No. of & $\begin{array}{l}\text { Number of } \\
\text { Disable }\end{array}$ & $\begin{array}{c}\% \text { of } \\
\text { Dis }\end{array}$ & Number of & $\begin{array}{c}\% \text { of } \\
\text { of }\end{array}$ \\
\hline 1 & Sitting/kneeling/standing & 10 & 51 & \multicolumn{2}{|c|}{$31(60.8 \%)$} & \multicolumn{2}{|c|}{$63(12.4 \%)$} \\
\hline 2 & Functions of the legs & 11 & 51 & \multirow{2}{*}{\multicolumn{2}{|c|}{$33(64.7 \%)$}} & \multicolumn{2}{|c|}{$114(20.3 \%)$} \\
\hline 3 & Functions of the arms & 6 & 51 & & & \multicolumn{2}{|c|}{$44(14.4 \%)$} \\
\hline 4 & Use of transportation & 3 & 51 & \multicolumn{2}{|c|}{$11(21.6 \%)$} & \multicolumn{2}{|c|}{$69(45.1 \%)$} \\
\hline 5 & Self-care & 9 & 51 & \multicolumn{2}{|c|}{$17(33.3 \%)$} & \multicolumn{2}{|c|}{$109(23.7 \%)$} \\
\hline 6 & Household tasks & 3 & 51 & \multicolumn{2}{|c|}{$4(7.8 \%)$} & \multicolumn{2}{|c|}{$65(42.5 \%)$} \\
\hline 7 & Leisure activities and sports & 11 & 51 & \multicolumn{2}{|c|}{$17(33.3 \%)$} & \multicolumn{2}{|c|}{$393(70.1 \%)$} \\
\hline & al Score & 53 & 51 & \multicolumn{2}{|c|}{$38(74.5 \%)$} & \multicolumn{2}{|c|}{$857(31.7 \%$} \\
\hline
\end{tabular}

$@$ No. of cases having normalization score less than 100 in a specific domain

\$Proportion of disabled for that specific domain from total cases i.e.51

*Calculated by summing invalids of all patients in a specific domain.

\#Proportion of sum of invalids from total items in each domain for all cases [\% of $c /(a \times b)]$ 
Table 3: Demographic distribution of all participants and correlation of PedHAL score

\begin{tabular}{|c|c|c|c|c|c|}
\hline Parameters & Frequency & Percentage & $\begin{array}{c}\text { Mean PedHDL } \\
\text { score }\end{array}$ & SD & $\begin{array}{c}\text { P value } \\
\text { (t test) }\end{array}$ \\
\hline \multicolumn{6}{|c|}{ Age } \\
\hline 4-5years & 6 & $11.8 \%$ & 93.02 & 10.95 & \multirow[t]{3}{*}{0.755} \\
\hline $6-9$ years & 21 & $41.2 \%$ & 86.29 & 20.99 & \\
\hline $10-14$ years & 24 & $47.1 \%$ & 84.01 & 32.26 & \\
\hline Total & 51 & $100.0 \%$ & 86.01 & 25.97 & \\
\hline \multicolumn{6}{|c|}{ BMI $(\mathrm{Kg} / \mathrm{m} 2)$} \\
\hline Underweight & 13 & $25.5 \%$ & 81.18 & 24.16 & \multirow{3}{*}{$\begin{array}{c}\text { UW Vs } \mathrm{NW}-\mathrm{p} \\
\text { value } 0.19 \\
\& \\
\text { OW Vs } \mathrm{NW}-\mathrm{p} \\
\text { value } 0.99\end{array}$} \\
\hline Normal Weight & 25 & $49.0 \%$ & 89.96 & 16.94 & \\
\hline Over weight + Obese & 13 & $25.5 \%$ & 90.56 & 13.27 & \\
\hline Total & 51 & $100 \%$ & 87.87 & 18.33 & \\
\hline \multicolumn{6}{|c|}{$\begin{array}{c}\text { UW = Underweight } \\
\mathrm{NW}=\text { Normal weight } \\
\mathrm{OW}=\text { Over weight }+ \text { Obese }\end{array}$} \\
\hline \multicolumn{6}{|c|}{ Education of the participants } \\
\hline Non school going & 8 & $15.7 \%$ & 83.10 & 21.77 & \multirow[t]{3}{*}{0.699} \\
\hline School going & 43 & $84.3 \%$ & 86.55 & 26.88 & \\
\hline Total & 51 & $100.0 \%$ & 86.01 & 25.97 & \\
\hline \multicolumn{6}{|c|}{ Father's Education } \\
\hline Primary or below & 11 & $21.6 \%$ & 95.95 & 7.01 & \multirow[t]{2}{*}{0.017} \\
\hline Secondary or above & 40 & $78.4 \%$ & 82.87 & 28.88 & \\
\hline Total & 51 & $100.0 \%$ & 86.01 & 25.97 & \\
\hline \multicolumn{6}{|c|}{ Mother's education } \\
\hline Primary or below & 27 & $52.9 \%$ & 88.65 & 15.412 & \multirow[t]{2}{*}{0.754} \\
\hline Secondary or above & 24 & $47.1 \%$ & 87.01 & 21.450 & \\
\hline Total & 51 & $100.0 \%$ & 86.01 & 25.97 & \\
\hline \multicolumn{6}{|c|}{ Type of Family } \\
\hline Nuclear & 42 & $82.4 \%$ & 84.64 & 28.10 & \multirow[t]{2}{*}{0.422} \\
\hline Joint & 9 & $17.6 \%$ & 92.39 & 10.69 & \\
\hline Total & 51 & $100.0 \%$ & 86.01 & 25.97 & \\
\hline \multicolumn{6}{|c|}{ Distance from home to hospital } \\
\hline$\leq 15 \mathrm{~km}$ & 38 & $74.5 \%$ & 87.46 & 17.953 & \multirow[t]{2}{*}{0.787} \\
\hline$>15 \mathrm{~km}$ & 13 & $25.5 \%$ & 89.08 & 20.086 & \\
\hline Total & 51 & $100.0 \%$ & 86.01 & 25.97 & \\
\hline \multicolumn{6}{|c|}{ Exercise Habit } \\
\hline Yes & 27 & $52.9 \%$ & 81.13 & 29.99 & \multirow[t]{2}{*}{0.148} \\
\hline No & 24 & $47.1 \%$ & 91.49 & 19.77 & \\
\hline Total & 51 & $100.0 \%$ & 86.01 & 25.97 & \\
\hline \multicolumn{6}{|c|}{ Frequency of exercise } \\
\hline Irregular & 10 & $37.0 \%$ & 91.08 & 18.60 & \multirow[t]{2}{*}{0.109} \\
\hline Regular & 17 & $63.0 \%$ & 75.85 & 35.08 & \\
\hline Total & 27 & $100.0 \%$ & 81.13 & 29.99 & \\
\hline \multicolumn{6}{|c|}{ Income per head per month (in Rs.) } \\
\hline$<834$ & 5 & $9.8 \%$ & 97.20 & 3.235 & \multirow[t]{5}{*}{0.019} \\
\hline $835-1670$ & 14 & $27.5 \%$ & 83.34 & 19.757 & \\
\hline $1671-278$ & 20 & $39.2 \%$ & 93.78 & 8.480 & \\
\hline $2785-5569$ & 9 & $17.6 \%$ & 85.80 & 21.245 & \\
\hline$>5570$ & 3 & $5.9 \%$ & 60.38 & 38.549 & \\
\hline Total & 51 & $100.0 \%$ & 86.01 & 25.97 & \\
\hline
\end{tabular}


Table 4: Distribution of the participants according to their medical and therapeutic parameters

\begin{tabular}{|c|c|c|c|c|c|}
\hline Parameters & Frequency & Percentage & Mean & SD & $\begin{array}{c}\text { P value } \\
\text { (t test) }\end{array}$ \\
\hline \multicolumn{6}{|c|}{ Age of Onset of symptoms } \\
\hline$<12$ months & 23 & $45.1 \%$ & 78.75 & 23.58 & \multirow[t]{2}{*}{0.001} \\
\hline$\geq 12$ months & 28 & $54.9 \%$ & 95.37 & 6.30 & \\
\hline Total & 51 & $100.0 \%$ & 87.87 & 18.32 & \\
\hline \multicolumn{6}{|c|}{ Severity of disease } \\
\hline Mild & 2 & $3.9 \%$ & 100.00 & 0.00 & \multirow[t]{3}{*}{0.38} \\
\hline Moderate & 4 & $7.8 \%$ & 74.17 & 49.47 & \\
\hline Severe & 45 & $88.2 \%$ & 86.44 & 24.04 & \\
\hline Total & 51 & $100.0 \%$ & 86.01 & 25.97 & \\
\hline \multicolumn{6}{|c|}{ Numbers of Bleed in last one year } \\
\hline Less than 6 & 43 & $84.3 \%$ & 91.02 & 15.26 & \multirow[t]{2}{*}{0.003} \\
\hline 6 or more & 8 & $15.7 \%$ & 70.94 & 24.74 & \\
\hline Total & 51 & $100.0 \%$ & 87.87 & 18.32 & \\
\hline \multicolumn{6}{|c|}{ Numbers of Bleed till date } \\
\hline 20 or less & 31 & $60.8 \%$ & 87.24 & 18.467 & \multirow[t]{2}{*}{0.76} \\
\hline More than 20 & 20 & $39.2 \%$ & 88.87 & 18.538 & \\
\hline Total & 51 & $100.0 \%$ & 86.01 & 25.97 & \\
\hline \multicolumn{6}{|c|}{ Family history of hemophilia } \\
\hline Present & 24 & $47.1 \%$ & 88.25 & 25.62 & \multirow[t]{2}{*}{0.567} \\
\hline Absent & 27 & $52.9 \%$ & 84.02 & 26.61 & \\
\hline Total & 51 & $100.0 \%$ & 86.01 & 25.97 & \\
\hline \multicolumn{6}{|c|}{ Treatment modalities } \\
\hline Factor 8 & 15 & $29.4 \%$ & 96.95 & 5.65 & \multirow[t]{2}{*}{0.004} \\
\hline Factor $8 \&$ Other & 36 & $70.6 \%$ & 81.45 & 29.64 & \\
\hline Total & 51 & $100.0 \%$ & 86.01 & 25.97 & \\
\hline
\end{tabular}

\section{Discussion}

The inherited disorders of blood coagulation present formidable problems top providers of health and social care with. The disorders are eminently treatable, even in their severest form. Untreated, they result in handicaps in early life, while proper treatment is expensive, inadequate treatment is even more so, both to the individual and to the community (Bhattacharya 1987). ${ }^{8}$

The incidence of hemophilia $\mathrm{A}$ is very low and it is easy to see how the disease can be overlooked in countries, like India, faced with major problems of malnutrition and infection. However ICMR Task Force $(1990)^{9}$ estimates that 1330 children are born every year and 500000 patients of hemophilia A are there of whom less than 5\% have access to medical facilities. The World Federation of Hemophilia (WFH) acknowledges the fact that the treatment is only presently available to around $20 \%$ of those with severe hemophilia in developed countries and the number is insignificant in developing countries (Jones 1991). ${ }^{10}$ The task is therefore to do everything possible to help persons with hemophilia in developing countries, arrange for the provision of effective therapy.

Studying of disability in hemophiliac and its correlation with number of bleeds and type of therapy (on demand vs prophylaxis) can help providers to make a more economic and beneficial prophylaxis strategies for the PWH. In this study it was found that 38 cases had disability in one or more domain. Highest number of disable was found in

functioning of Leg (domain 2) which was followed by domain 1, i.e, Sitting/kneeling/standing function. Similar study using PedHAL score has been done on Romanian children who are under on demand treatment like Indian scenario. In this study the PedHAL sum scores ranged from 19.2 to 99 with median score of 83.5 (IQR 47.9-90.5). This suggests that functional status of current study population is better compared to Romanian hemophilia patients.

As per Kar A et al study disability was widely prevalent as only 9 of 148 patients were free from disability, severe disability in the 25+, 13-24 age group 5-12 age group was $79 \%, 68 \%$ and $38 \%$ respectively. In the pre-adolescent age group of 5-12 years 79\% of boys showed moderate to severe disability, $88 \%$ of all patients had severe to moderately affected knee joint. This also suggest that squatting was affected which is one major daily routine of Indian patients. ${ }^{11}$ Also it was found that the some of the children had not been allowed or had ever performed the activity, such items were considered invalid. In present study the highest invalids were found in Leisure activities and sports (domain 7) and the least were in domain 1.

The correlation of various parameters and the PedHAL was done in this study. Form the result it is found that PedHAL score decreases with age indicate that disability increases with age but the difference was statistically insignificant ( $\mathrm{p}>0.05$ ) According to Kar et all study, in the 
age group of 5 to 12 years, only 15 per cent of patients were disability-free. ${ }^{11}$ Current study gives the degree of disability using Ped HAL score in above studied age groups. According to a study by Kar A \& Mirkazemi R the extent of disability is proportional to age. The number of affected joints increased progressively with age, so that by the age of 25 years, mobility of all patients were affected. ${ }^{11}$ In present study there was no any significant correlation found in the score among the participant with different BMI index. CDC data have shown that in addition to increasing severity of hemophilia, overweight, as measured by body mass index (BMI), is strongly associated with joint mobility limitations. Excess body adiposity accelerates joint mobility loss in weight bearing joints particularly among those with severe hemophilia. A study suggest that body weight control and effective treatment of bleeds should be implemented together to achieve better joint ROM outcomes in males with hemophilia. ${ }^{12}$

According to Kar A et al study conducted on 148 hemophilia patients across India $36.5 \%$ discontinued education of which $96.3 \%$ ascribed it to health related reasons also proportion of severe and moderate disability was $69.1 \%$ and $30.9 \%$ in that study group. ${ }^{11}$ In present study, PedHAL score was not correlated with the education of patients. Children with Hemophilia $(\mathrm{CWH})$ face restrictions at a very early age as parents try to protect them from bleeds. This stress and isolation becomes severe, when frequent bleeds lead to disability. This disability in turns feeds on the realization of being a burden to both family and society. Due to overprotection by the parents, irregular schooling, unemployment and loneliness, all Parents and Children Hemophilia suffers from Psycho - Social problems. Also the education of parents also make much difference in the PedHAL score of the patients. In present study it was found that, PedHAL score was not correlated with the type of family patient. But Kar et al study shows that disability was significantly associated with number of family members and not with type of family. ${ }^{11}$

Long term outcome of patients with joint bleed is best if therapy is administered within two hours of onset of joint bleed. In current study discrepancy of disability score with distance of treatment centre could be due to lack of awareness about importance of early factor administration in those residing $<15 \mathrm{~km}$ and modes of transport used for reaching the centre by the two groups under study. PedHAL score was not correlated with the distance of residence of the patients from home to hospital.

A low PedHAL score i.e greater disability is expected in those who do not exercise. In current study as shown in the above table mean PedHAL Score was higher in those did not do (91.49) compare to cases who do exercise (81.13). This discrepancy could be due to infrequent and improper methods of exercise practiced in patients with hemophilia. Joint exercises by expert physiotherapist is a part of comprehensive care in hemophiliacs, that helps both in strengthening of joints and prevent bleeds and to reduce impairment in affected joints. In current study disability was more in those on regular exercise; this could be due to improper manner of exercise.

In present study, PedHAL score was increasing with decrease in income. This indicates that PedHAL score was decreases (disability increases) as the income rises. This discrepancy could be due to small sample size. Factor therapy being costly is not affordable for an average Indian family of hemophilia patient. In developing country like India where treatment modality is on demand mainly and usually cryoprecipitate or recombinant factor made available by health providers; income of family matters less. PedHAL Score was lower in cases developed symptoms before the age of 12 months (78.75) compares to cases that developed it later (95.37). A lower PedHAL score means higher disability may be because early onset of symptoms is associated with greater morbidity and more joint damage. However there has been no other study that compares degree of disability with age of onset. The correlation between PedHAL score and the severity of disease was statistically not significant. ( $p>0.05$ )

The PedHAL score was significantly lower with 6 or more bleed per year. As discussed above higher number of bleeds is associated with more joint damage due to recurrent inflammation. In current study it was observed that those patients with more than 6 bleeds a year had more disability (lower PedHAL score) and it was also statistically significant. The long-term consequences of repeated joint bleeds with sub-optimal treatment lead to the development of chronic and progressive joint damage and disability. ${ }^{13}$ Only one study by Kar et $\mathrm{al}^{11}$ has measured the prevalence of disability. This study, conducted at five centres across the country showed that of the 148 patients with severe hemophilia A, only nine were free of disability. Patients with moderate and severe hemophilia have higher frequency of clinical and subclinical bleeds thus more prone to arthropathy than mildly severity patients.

In Kar et al study, disability was significantly associated with family history of hemophilia $(\mathrm{P}=0.004)$ with more disability free patients belonging to families with another affected person. But in present study, PedHAL score was not correlated with the family history of hemophilia. ${ }^{11}$ PedHAL Score was higher in cases receiving only factor $8(96.95)$ compare to cases receiving factor 8 with other drugs (81.45) and difference was statistically significant $(\mathrm{p}<0.01)$ indicates that PedHAL score was higher in cases taking only factor 8 . Thus disability was lesser in hemophilia patients using factor 8 as only modality of treatment. Kar A et al. study disability data for 41 patients who had primarily used CFCs were compared with 62 patients who had primarily used cryoprecipitate also showed that significant difference in physical disability scores between these two groups of patients $(\mathrm{P}=0.000)$, with more disabled patients belonging to the group primarily using cryoprecipitate. ${ }^{11}$

In this study the mean PedHAL score is not associated with age of the patient; nutritional status of patient; education of patient; severity of disease; number of bleeds till date; distance from home to hospital; habit of exercise; 
regularity of exercise; type of family of patient; family history of hemophilia; education of patients' mother; and family income of the patient. Early onset of symptoms is associated with lower PedHAL score and thus higher disability. Cases having 6 or more bleed per year had lower PedHAL score and thus higher disability. Higher PedHAL score and thus lower disability is associated with cases taking only factor 8 compared to cases taking other medication along with factor 8 .

The main weak aspect of this study is the size of the study population. One can find the more accurate result and by increasing the study population.

\section{Source of funding}

None.

\section{Conflict of interest}

None.

\section{References}

1. Klinge J. Hemophilia A-from basic science to clinical practice. Seminars in thrombosis and hemostasis, 2002;28(3):309-22.

2. Bolton-Maggs, P.H. and K.J. Pasi, Hemophilias A and B. Lancet 2003;361(9371):1801-9.

3. World Federation of Hemophilia, Report on the Annual Global Survey 2009, p. 14.

4. World Federation of Hemophilia, Global Incidence and Prevalence Rates Approved by the President's Strategic Council. 2003.

5. Evatt B L. The natural evolution of hemophilia care: developing and sustaining comprehensive care globally. Hemophilia: the official $J$ World Federation Hemophilia 2006;12(Suppl 3):13-21.
6. World health organisation. ICF: international classification of functioning disability and health. Geneva: world health organisation 2001.

7. Groen W, Van der Net J, Helders P, Fischer K. Paediatric Hemophilia Activities List (PedHAL) v0.11. Available from: http://elearning.wfh.org/resource/hemophilia-activities-listpediatric-pedhal/ [Accessed 26 Nov 2019.]

8. Bhattacharya DK. A study of bleeding episodes of hemophilia A. Cal Med J 1987;84:1-8.

9. ICMR Task Force. Collaborative Study on Hemophilia, New Delhi, Indian Council of Medical Research, 1990.

10. Jones PK1, Ratnoff OD. The changing prognosis of classic hemophilia (factor VIII "deficiency"). Ann Intern Med 1991;114(8):641-8.

11. Kar A, Mirkazemi R, Singh P, Potnis-Lele M, Lohade S, Lalwani A, et al. Disability in Indian patients with hemophilia. Hemophilia 2007;13:398-404.

12. Soucie JM, Wang C, Siddiqi A, Kulkarni R, Recht M, Konkle BA; Hemophilia Treatment Center Network. The longitudinal effect of body adiposity on joint mobility in young males with Hemophilia A. Hemophilia 2011;17:196-203.

13. Nair AP, Jijina F, Ghosh K, Madkaikar M, Shrikhande M, Nema M. Osteoporosis in young hemophiliacs from western India. Am J Hematol 2007;82:453-7.

How to cite this article: Shah VB, Mehta $\mathrm{K}$, Agrawal S. Study of the degree of disability in children suffering from Hemophilia- A using PedHAL score in south Gujarat area. Int J Med Paediatr Oncol 2019;5(4):13845. 